\title{
Aditivo orgánico en raciones para pacú (Piaractus mesopotamicus) en período reproductivo. Efecto sobre la recuperación de lesiones
}

\author{
Della Rosa, P.; Hernández, D.R.; Roux, J.P.; Santinón, J.J.; Sánchez, S. \\ Instituto de Ictiología del Nordeste, Facultad de Ciencias Veterinarias, UNNE, Sargento Cabral 2139, \\ Corrientes (3400), Argentina. E-mail: dhernandez@vet.unne.edu.ar.
}

\begin{abstract}
Resumen
Della Rosa, P.; Hernández, D.R.; Roux, J.P.; Santinón, J.J.; Sánchez, S.: Aditivo orgánico en raciones para pacú (Piaractus mesopotamicus) en período reproductivo. Efecto sobre la recuperación de lesiones. Rev. vet. 23: 2, 81-85, 2012. El objetivo del presente trabajo fue evaluar la recuperación de lesiones en reproductores de pacú (Piaractus mesopotamicus) utilizando un reparador metabólico a base de flavonoides ofrecido antes o después de las maniobras reproductivas (inducción y desove) en el alimento. Se utilizaron 39 ejemplares de pacú, los que se distribuyeron aleatoriamente en tres grupos según el tipo de alimento ofrecido: (G1) ración comercial; (G2) ración comercial hasta las maniobras reproductivas donde cambia a ración comercial adicionada con $2 \mathrm{~g} / \mathrm{kg}$ de Flavoxin ${ }^{\circledR}$; y (G3) ración comercial adicionada con $2 \mathrm{~g} / \mathrm{kg}$ de Flavoxin ${ }^{\circledR}$ durante todo el período experimental. El ensayo tuvo una duración de 69 días, período en el cual se realizaron biopsias ováricas como seguimiento de la maduración gonadal. Durante el período previo a la hipofisación y desove o espermiación, los reproductores mostraron buen estado general. Por el contrario, e inmediatamente posterior a las maniobras reproductivas, se observó un alto número de lesiones en tegumento y aletas en todos los ejemplares correspondientes al G1 y G2, destacándose el reducido número de lesiones en G3, el cual se diferenció estadísticamente $(p<0,05)$. En G3 no se observaron lesiones posteriormente a los 9 días de las maniobras reproductivas, mientras que en G1 y G2 las mismas aún se encontraron presentes al final de la experiencia. Asimismo, al finalizar la experiencia el porcentaje de supervivencia del G2 fue el más bajo (60\%), diferenciándose de G1 y G3 $(\mathrm{p}<0,05)$. Estos resultados demuestran que la utilización de $2 \mathrm{~g} / \mathrm{kg}$ de Flavoxin ${ }^{\circledR}$ adicionado a raciones para reproductores de $P$. mesopotamicus, previamente a la realización de las maniobras reproductivas, permite una pronta recuperación de las lesiones post-desove o espermiación, mejorando el estado sanitario de los mismos.
\end{abstract}

Palabras clave: Piaractus mesopotamicus, prebiótico, flavonoides, reproducción, lesiones.

\begin{abstract}
Della Rosa, P.; Hernández, D.R.; Roux, J.P.; Santinón, J.J.; Sánchez, S.: Organic additive in rations for pacu (Piaractus mesopotamicus) during the reproductive period. Effect on recovery from injuries. Rev. vet. 23: 2, 81-85, 2012. The aim of this study was to evaluate the recovery from injuries in broodstocks of pacu (Piaractus mesopotamicus) using commercial flavonoids added to the food before or after hormonal manipulations (induction of final oocyte maturation and spawning). Thirty nine adults of pacu were randomized into three groups according to the type of food offered: (G1) commercial diet, (G2) commercial diet until reproductive manipulations and then changing to commercial diet plus $2 \mathrm{~g} / \mathrm{kg}$ of Flavoxin $^{\circledR}$, and (G3) commercial diet supplemented with $2 \mathrm{~g} / \mathrm{kg}$ of Flavoxin ${ }^{\circledR}$ throughout the experimental period. The experiment lasted 69 days and during this period of time ovarian biopsies were performed to follow up gonadal maturation. Prior to hypophysation, the broodstocks showed good general condition. However, immediately after reproductive procedures, it was observed a high number of injuries in tegument and fins in all the fish from G1 and G2, and low number of injuries in G3, which was statistically different $(p<0.05)$. In G3, nine days after reproductive procedures no lesions were observed, whereas in G1 and G2, injuries were still present until the end of the experiment. The lowest survival rate was observed in G2 $(60 \%)$, differing from G1 and G3 $(\mathrm{p}<0.05)$. These results demonstrate that the use of $2 \mathrm{~g} /$ $\mathrm{kg}$ Flavoxin ${ }^{\circledR}$ added to the ration for P. mesopotamicus broodstocks prior to reproductive procedures allows a quick recovery from post-spawning injuries, thus improving health status.
\end{abstract}

Key words: Piaractus mesopotamicus, prebiotic, flavonoids, reproduction, injuries. 


\section{INTRODUCCIÓN}

El proceso reproductivo en peces teleósteos está controlado por el eje hipotálamo-hipófisis-gonadal ${ }^{24}$, siendo activado por factores externos como la temperatura del agua y el fotoperíodo ${ }^{6,21}$. Sin embargo, la mayoría de los peces en cautiverio no desovan por bloqueos a diferentes niveles del proceso de maduración gonadal ${ }^{32}$, en particular en especies migradoras como el pacú ${ }^{17}$.

El estrés inducido por la cautividad como resultado de la combinación de diferentes condiciones adversas, entre ellas, deficiencias alimenticias, mala calidad de agua, alta densidad de reproductores y otras, repercuten en una disfunción reproductiva ${ }^{29}$, y como resultado se produce un fallo en la liberación de la gonadotropina pituitaria luteinizante ${ }^{2}$. Así, la inducción hormonal es una herramienta que permite estimular los procesos reproductivos de maduración ovocitaria final, ovulación y posterior desove ${ }^{19}$, donde la hipofisación es una de las técnicas de inducción más utilizada ${ }^{24}$.

Sin embargo, esta práctica de manejo lleva implícita la manipulación periódica de los reproductores originando diversas lesiones principalmente en tegumento. El daño físico y el estrés fisiológico de la captura, manipulación, transporte, inyección hormonal y el masaje abdominal para forzar al desove tiene un efecto negativo en los reproductores dando lugar a la presentación de diversas patologías ${ }^{9}$. Varios productos terapéuticos se utilizan para prevenir o tratar la aparición de enfermedades luego de las maniobras reproductivas, siendo los antibióticos uno de los más utilizados ${ }^{7}$. A pesar de estas medidas, un porcentaje de peces muere ${ }^{31}$ ocasionando la pérdida del reproductor que demandó mucho esfuerzo en su mantenimiento y selección.

Numerosos estudios se centraron en el uso de suplementos alimenticios como herramienta para promover el bienestar de los peces cultivados. Dentro de las alternativas más utilizadas se incluye la adición de microorganismos benéficos, prebióticos, acidificantes orgánicos, extractos de levaduras, entre otros. Todos ellos se orientaron a identificar alternativas que favorezcan la salud ${ }^{16}$ y la capacidad de resistir enfermedades en reemplazo de los quimioterápicos y antimicrobianos ${ }^{3,15,28}$.

Los prebióticos flavonoides son aditivos alimentarios naturales presentes en plantas que poseen efectos antioxidantes, antiinflamatorios y anticarcinogénicos ${ }^{18,25}$. Muchos estudios en seres humanos demostraron que los flavonoides pueden utilizarse terapéuticamente con acciones antioxidantes al reducir los radicales libres que inducen injurias en los tejidos $5,11,18,20,22$. Además, es conocido que los flavonoides redu- cen o inhiben el crecimiento de muchos microorganismos y de sus toxinas ${ }^{11,20}$.

Si bien el uso de prebióticos en acuicultura se encuentra en aumento ${ }^{26}$ la información referida a la utilización de estos productos en dietas para reproductores es escasa. En el presente trabajo se propone evaluar el efecto de la adición de un aditivo orgánico en dietas para reproductores de pacú sobre la recuperación de lesiones ocasionadas por las maniobras reproductivas (inducción y desove).

\section{MATERIAL Y MÉTODOS}

Reproductores y alimentación. Se utilizaron 39 ejemplares adultos de pacú (machos y hembras) con signos externos de madurez sexual (según Da Silva ${ }^{12}$ ), obtenidos de la Estación Experimental Agropecuaria Corrientes (Corrientes, Argentina). El ensayo se realizó en las instalaciones del Instituto de Ictiología del Nordeste (INICNE) de la Facultad de Ciencias Veterinarias, UNNE (Corrientes, Argentina). Los mismos fueron distribuidos aleatoriamente en estanques de mampostería de $4 \mathrm{~m}^{3}$ en tres grupos iguales según el tipo de alimento ofrecido: (G1) ración comercial control (280 g/kg de proteínas y $120 \mathrm{~g} / \mathrm{kg}$ de lípidos); (G2) ración comercial hasta las maniobras reproductivas donde cambia a ración comercial adicionada con $2 \mathrm{~g} / \mathrm{kg}$ de Flavoxin ${ }^{\circledR}$ (Vetanco S.A., Buenos Aires, Argentina); y (G3) ración comercial adicionada con $2 \mathrm{~g} / \mathrm{kg}$ de Flavoxin ${ }^{\circledR}$ durante todo el período experimental (Figura 1).

El ensayo tuvo una duración de 69 días, período en el cual los ejemplares se mantuvieron bajo un fotoperíodo natural y fueron alimentados una vez al día a razón del 5\% de la biomasa. Los alimentos fueron elaborados mediante el molido de la dieta comercial y posterior mezclado con el aditivo. Seguidamente se adicionó agua $(400 \mathrm{~g} / \mathrm{kg})$ para formar una pasta blanda. La pasta obtenida fue pasada por una peletizadora para obtener pellets de $4 \mathrm{~mm}$ de diámetro, los que fueron

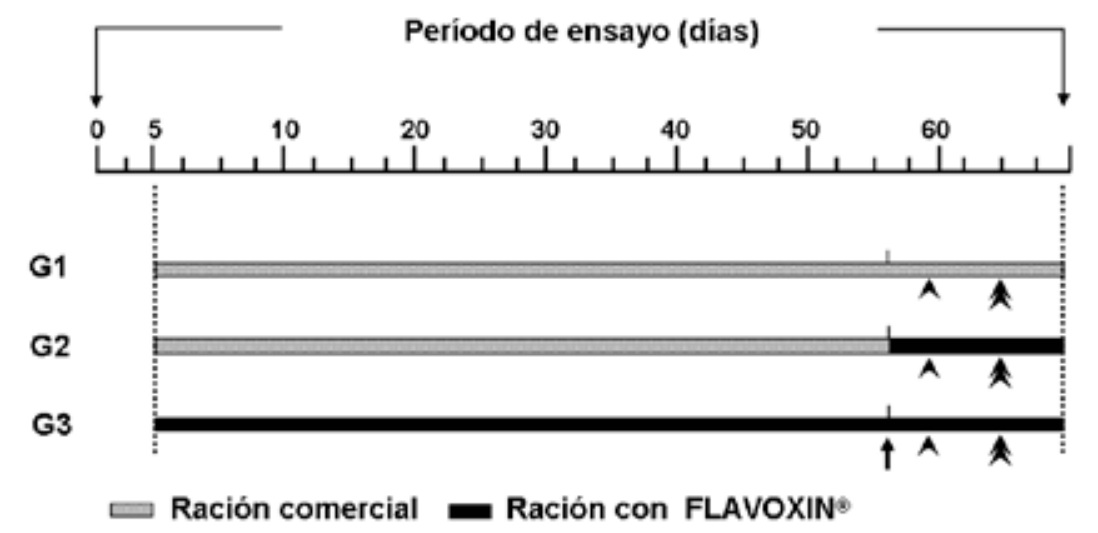

Figura 1. Esquema de los diferentes grupos evaluados. La flecha indica el momento de inducción hormonal y desove en laboratorio; la cabeza de flecha indica el momento en que son transferidos nuevamente a estanque y primera observación de lesiones; la doble cabeza de flecha indica la segunda evaluación de lesiones. 
secados en estufa a $40^{\circ} \mathrm{C}$ durante $48 \mathrm{~h}$ y conservados en freezer a $-18^{\circ} \mathrm{C}$ durante el período experimental. La dieta control fue sometida al mismo tratamiento sin el agregado del aditivo.

Seguimiento de reproductores e inducción hormonal. Durante el transcurso del ensayo se llevaron a cabo dos biopsias ováricas a las hembras de cada grupo para verificar el estado de maduración gonadal. Una vez alcanzado un mínimo de $45 \%$ de oocitos con núcleo excéntrico en todos los tratamientos (56 días de ensayo) los peces se trasladaron al laboratorio de reproducción y se alojaron en tanques de fibra de vidrio de 300 litros, con aireación forzada y recambio de agua a razón de 60 litros/hora. En ese momento, se procedió a aplicar la técnica de la inducción con extractos de hipófisis según el protocolo propuesto por Da Silva ${ }^{12}$, para luego realizar el desove mediante presión abdominal. Concluidas las maniobras reproductivas, los ejemplares fueron mantenidos en laboratorio por 72 horas y, posteriormente, transferidos a estanques permaneciendo diez días hasta el final del ensayo.

Medición de parámetros y análisis estadísticos. La presencia de lesiones post-reproducción fue registrada en tres oportunidades, (i) al momento de transferir los reproductores a estanques $(72 \mathrm{~h}$ posteriores a las maniobras reproductivas), (ii) luego de 6 días en estanques (9 días posteriores a las maniobras reproductivas) y, (iii) luego de 10 días en estanques, al final de la experiencia (13 días posteriores a las maniobras reproductivas) (Figura 1), cuantificando el número de ejemplares con lesiones (en cabeza, cuerpo o aletas) y sin lesiones. Además, se consideraron los animales muertos entre cada período.

La supervivencia (S), fue estimada al final del ensayo de la siguiente manera: $\mathrm{S}(\%)=100\left(N_{f} N_{i}^{-l}\right)$, donde $N_{f}=$ número de peces al final del ensayo y $N_{i}=$ número de peces al inicio del ensayo. Diariamente se realizó la medición de la temperatura del agua de los estanques, y con frecuencia semanal, se tomaron los siguientes pa-

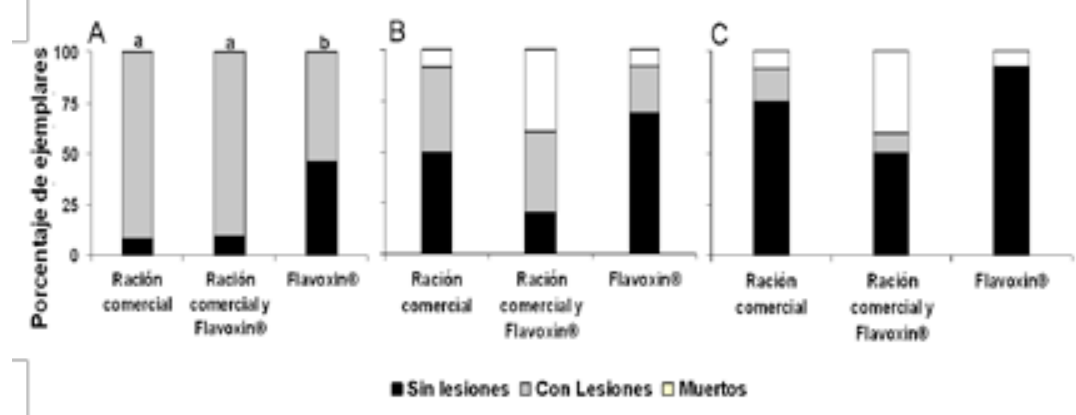

Figura 2. Porcentaje de ejemplares de P. mesopotamicus con lesiones posteriores a la inducción y desove o espermiación. (A), $72 \mathrm{~h}$ posteriores a las maniobras reproductivas (transferidos a estanques). (B), luego de 6 días en estanques ( 9 días posteriores a las maniobras reproductivas). (C), al final de la experiencia (13 días posteriores a las maniobras reproductivas). Letras diferentes indican diferencias significativas entre grupos $(\mathrm{p}<0,05)$.
Tabla 1. Parámetros de calidad de agua durante el período experimental.

\begin{tabular}{lr}
\hline parámetro & promedio $\pm \mathrm{DE}$ \\
\hline temperatura ${ }^{\circ} \mathrm{C}$ & $26 \pm 0,12$ \\
$\mathrm{pH}$ & $6,82 \pm 0,13$ \\
oxígeno $(\%)$ & $113,9 \pm 3,65$ \\
oxígeno $(\mathrm{mg} / \mathrm{l})$ & $8,65 \pm 0,53$ \\
conductividad $(\mu \mathrm{S} / \mathrm{cm})$ & $124 \pm 1,14$ \\
\hline
\end{tabular}

rámetros de calidad de agua: $\mathrm{pH}$, conductividad y oxígeno disuelto (mg/l y \% de saturación). El análisis de los datos se realizó mediante el método no paramétrico de Chi-cuadrado $(\mathrm{p}<0.05)$.

\section{RESULTADOS Y DISCUSIÓN}

Los parámetros físicos y químicos del agua se mantuvieron dentro de un rango de valores adecuados para la especie (Tabla 1). Durante el transcurso del ensayo previamente a la inducción al desove, los ejemplares no presentaron lesiones. Sin embargo, a las $72 \mathrm{~h}$ posteriores a las maniobras reproductivas (59 días de ensayo) se observó que los ejemplares de los tres grupos presentaron lesiones distribuidas en diferentes partes del cuerpo, principalmente en la zona de la cabeza, aletas y región abdominal. Esta última es la que se vio más comprometida, probablemente por la presión ejercida por el masaje abdominal para forzar el desove. En G3 se encontró un bajo número de animales con lesiones, inferior al observado en G1 y G2 $(p<0,05)$, sin diferencias significativas entre estos últimos grupos $(p>0,05)$ (Figura 2 A). No se observaron animales muertos en este período.

Transcurridos seis días en estanques, murieron algunos ejemplares en todos los grupos (las muertes ocurrieron entre el $5^{\circ}$ y $6^{\circ}$ día), aunque la mayor mortalidad se observó en el grupo 2, al tiempo que se constató una recuperación de las lesiones en los peces sobrevivientes (Figura $2 \mathrm{~B}$ ). Al final de la experiencia, todavía pudieron observarse algunas lesiones leves en los ejemplares de los grupos $1 \mathrm{y}$ 2, mientras que los peces del grupo 3 ya no presentaron lesiones (Figura 2 C). Más allá de las diferencias mencionadas en cuanto a la evolución de las lesiones en los diferentes grupos, el análisis estadístico no permitió detectar diferencias significativas entre los mismos al considerar el número de ejemplares con lesiones luego de 6 o 10 días en estanques ( $p>0,05)$.

En cuanto al porcentaje de supervivencia, al final de la experiencia se constató que los grupos 1 y 3 presentaron valores cercanos al 92\%, mientras que G2 finalizó 
con $60 \%$, diferenciándose significativamente del resto $(\mathrm{p}<0,05)$ (Figura 3). La dieta y el estado nutricional son factores fundamentales para la explotación piscícola, los que resultan determinantes del estado fisiológico de los animales, tanto de manera directa por su influencia sobre el nivel energético, como de manera indirecta por su influencia sobre la resistencia al estrés ${ }^{8}$. Se afirma que una hembra bajo condiciones de estrés, puede seleccionar entre asignar energía para el mantenimiento o para la reproducción ${ }^{29}$. Sin embargo, en la mayoría de las especies bajo condiciones de cultivo, los reproductores no tienen posibilidad de elegir su estrategia reproductiva, siendo inducidos artificialmente sin haber alcanzado un desarrollo óptimo de madurez sexual o bien son utilizados en diferentes momentos dentro de la estación reproductiva, aumentando los riesgos de muerte.

En el presente trabajo se pudieron observar lesiones en todos los grupos posteriormente a las maniobras reproductivas. Otro estudio realizado en lenguado $(\mathrm{Pa}$ ralichthys orbignyanus) se registró un elevado número de lesiones en todo el cuerpo debido a la manipulación repetitiva ocasionada por el masaje abdominal luego de la inducción hormonal ${ }^{4}$. Asimismo, fueron observadas lesiones en lubina (Dicentrarchus labrax) luego de manipulaciones sucesivas relacionadas con los tratamientos hormonales en los tanques de reproducción ${ }^{14}$. Por el contrario, la selección de reproductores de pejerrey (Odontesthes bonariensis) por simple inspección visual del abdomen y ancho del poro genital evitaron las lesiones ocasionadas por manipulación y biopsias ováricas ${ }^{23}$. Además, la utilización de implantes de hormona liberadora de gonadotropina $(\mathrm{GnRH})$ reduce considerablemente el estrés en los reproductores ${ }^{23}$.

En nuestro trabajo observamos que la incorporación de flavonoides en la dieta influencia favorablemente la condición sanitaria de los reproductores de pacú en términos de recuperación de lesiones post-reproductivas en cautiverio. Los flavonoides son comúnmente utilizados como aditivos en raciones para peces y crustáceos con el objeto de mejorar el estado sanitario o la resistencia a patógenos ${ }^{1,10,27}$. En lenguados (Paralichthys olivaceus) se halló una mejora en la función inmune contra el estrés externo en ejemplares alimentados con quercetina como flavonoide a razón $5 \mathrm{~g} / \mathrm{kg}$ comparado con un alimento control, observándose un efecto hipocolesterolémico, antioxidante y aumento de la actividad lisozima ${ }^{30}$.

En el presente trabajo se observaron diferencias en la recuperación de lesiones entre el grupo 3 respecto de los grupos 1 y 2 . Si bien los animales del grupo 2 recibieron flavonoides en la ración, lo hicieron posteriormente a la inducción hormonal donde los peces no suelen consumir alimentos hasta varios días después, posiblemente por el estrés ocasionado. Este reducido tiempo (hasta trece días post-inducción) pudo no haber sido suficiente para obtener un efecto beneficioso de los flavonoides.

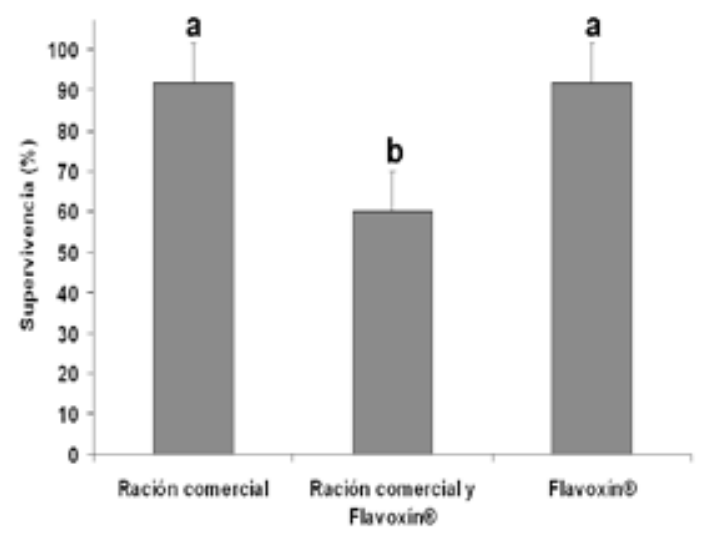

Figura 3. Porcentaje de supervivencia de reproductores de P. mesopotamicus al final de la experiencia (13 días posteriores a las maniobras reproductivas). Letras diferentes indican diferencias significativas entre gru$\operatorname{pos}(\mathrm{p}<0,05)$.

Los flavonoides poseen excelentes propiedades de quelación del hierro y otros metales de transición, lo que les confiere una gran capacidad antioxidante y protegen a los tejidos frente al daño oxidativo ${ }^{22}$. En el presente ensayo, no se observaron diferencias en el porcentaje de supervivencia en contraste con un tratamiento control. En lubina (D. labrax) luego de manipulaciones sucesivas relacionadas con los tratamientos hormonales en los tanques de reproducción se observó una mortalidad de hasta un 5\% por año y en algunos casos hasta un $100 \%$ en reproductores de menor tamaño ${ }^{14}$. Evidentemente, la búsqueda de una correcta adaptación de los animales a las condiciones de cultivo es una de las opciones a seguir ${ }^{13}$. Cuanto mejor es la adaptación, tanto mejor serán las posibilidades del mantenimiento en cautividad, el crecimiento y la reproducción de la especie ${ }^{29}$.

Estos resultados demuestran que la utilización de 2 $\mathrm{g} / \mathrm{kg}$ de Flavoxin ${ }^{\circledR}$ adicionado a raciones para reproductores de P. mesopotamicus, previamente a la realización de las maniobras reproductivas, permite una pronta recuperación de las lesiones post-desove, mejorando el estado sanitario de los mismos.

\section{REFERENCIAS}

1. Ardó L, Yin G, Xu P, Váradi L, Szigeti G, Jeney Z, Jeney G. 2008. Chinese herbs (Astragalus membranaceus and Lonicera japonica) and boron enhance the non-specific immune response of Nile tilapia (Oreochromis niloticus) and resistance against Aeromonas hydrophila. Aquaculture 275: 26-33.

2. Babin PJ, Cerdà J, Lubzens E. 2007. The fish oocyte. From basic studies to biotechnological applications, Springer, The Netherlands, $510 \mathrm{p}$.

3. Balcázar JL, De Blas I, Zarzuela-Ruiz I, Cunningham D, Vendrell D, Múzquiz JL. 2006. The role of probiotics in aquaculture. Vet Microbiol 114: 173-186. 
4. Bambill GA, Oka M, Radonić M, López AV, Müller MI, Boccanfuso JJ, Bianca FA. 2006. Broodstock management and induced spawning of flounder Paralichthys orbignyanus (Valenciennes, 1839) under a closed recirculated system. Rev Biol Mar Ocean 41: 45-55.

5. Barreca D, Lagana G, Tellone E, Ficarra S, Leuzzi U, Galtieri A, Bellocco E. 2009. Influences of flavonoids on erythrocyte membrane and metabolic implication through anionic exchange modulation. J Membrane Biol 230: 163171.

6. Bromage N, Porter M, Randall C. 2001. The environmental regulation of maturation in farmed finfish with special reference to the role of photoperiod and melation. Aquaculture 197: 63-98.

7. Cabello FC. 2004. Antibióticos y acuicultura en Chile: Consecuencias para la salud humana y animal. Rev Méd Chile 132: 1001-1006.

8. Canario A, Condeça J, Power DM, Ingleton PM. 1998. The effect of stocking density on growth in the gilthead sea-bream, Sparus aurata (L.). Aquaculture Res 29: 177181.

9. Carnevia D. 1993. Enfermedades de los peces ornamentales, Ed. Agro-Vet, Buenos Aires, 319 p.

10. Citarasu T. 2010. Herbal biomedicines: a new opportunity for aquaculture industry. Aquaculture Intern 18: 403-414.

11. Cushnie TP, Lamb AJ. 2005. Antimicrobial activity of flavonoids. Int J Antimicr Agents 26: 343-356.

12. Da Silva AB, Vinatea JE, Alcantara F. 1988 Manual de reproducción de peces colossoma (pacú y tambaqui), Tarapoto, San Martín (Perú), 95 p.

13. Davis LE, Okihiro MS, Hinton DE. 2002. Effects of husbandry practices, gender, and normal physiological variation on growth and reproduction of Japanese medaka, Oryzias latipes. Acuatic Toxicol 60: 185-201.

14. Devauchelle N, Coves D. 1988. Sea bass (Dicentrarchus labrax) reproduction in captivity: gametogenesis and spawning. Aquat Libing Resour 1: 215-222.

15. Fritts CA, Waldroup PW. 2003. Evaluation of Bio-Mos mannan oligosaccharide as a replacement for growth promoting antibiotics in diets of turkeys. Int J Poultry Sci 2: 19-22.

16. Gatesoupe FJ. 2007. Live yeasts in the gut: natural occurrence, dietary introduction, and their effects on fish health and development. Aquaculture 267: 20-30.

17. Iseki KK, Negrao JA. 2004. Variação sazonal do ciclo reprodutivo de fêmeas de pacu, Piaractus mesopotamicus (Teleostei, Characiformes, Characidae). Rev Ciências Vet 2: 188-193.

18. Knekt P, Jarvinen R, Seppanen R, Helibvaara M, Teppo L, Pukkala E, Aroma A. 1997. Dietary flavonoids and the risk of lung cancer and other malignant neoplasms. $\mathrm{Am}$ J Epidem 146: 223-230.
19. Lam TJ. 1982. Applications of endocrinology to fish culture. Can J Fish Aquat Sci 39: 111-137.

20. Lee KA, Moon SH, Kim KT, Mendonca AF, Paik HD. 2010. Antimicrobial effects of various flavonoids on Escherichia coli $\mathrm{O} 157: \mathrm{H} 7$ cell growth and lipopolysaccharide production. Food Sci Biotechnol 19: 257-261.

21. Lowe-McConnel RH. 1999. Estudos ecológicos de comunidades de peixes tropicais, Edit Univ São Paulo, São Paulo, Brasil, $534 \mathrm{p}$.

22. Martínez-Florez S, Gonzáles-Gallego J, Culebras JM, Tuñón MJ. 2002. Los flavonoides: propiedades y acciones antioxidantes. Nutr Hospit 17: 271-278.

23. Miranda LA, Somoza GM. 2009. Spawning induction of pejerrey Odontesthes bonariensis in captivity using sustained-release gonadotropin releasing hormone agonist implants. Aquaculture Res 41: 129-134.

24. Mylonas CC, Fostier A, Zanuy S. 2010. Broodstock management and hormonal manipulations of fish reproduction. Gen \& Comp Endocr 165: 516-534.

25. Nowakowska Z. 2007. A review of anti-infective and antiinflammatory chalcones. Eur J Med Chem 42: 125-137.

26. Ringø E, Olsen RE, Gifstad TØ, Dalmo RA, Amlund H, Hemre GI, Bakke AM. 2010. Prebiotics in aquaculture: a review. Aquaculture Nutr 16: 117-136.

27. Saera-Vila A, Benedito-Palos L, Sitjà-Bobadilla A, Nácher-Mestre J, Serrano R, Kaushik S, Pérez-Sánchez J. 2009. Assessment of the health and antioxidant trade-off in gilthead sea bream (Sparus aurata L.) fed alternative diets with low levels of contaminants. Aquaculture 296: 87-95.

28. Salinas I, Abelli L, Bertoni F, Picchietti S, Roque A, Furones D, Cuesta A, Meseguer J, Esteban MÁ. 2008. Monospecies and multispecies probiotic formulations produce different systemic and local immunostimulatory effects in the gilthead seabream (Sparus aurata L.). Fish \& Shellfish Imm 25: 114-123.

29. Schreck CB, Contreras-Sanchez W, Fitzpatrick MS. 2001. Effects of stress on fish reproduction, gamete quality, and progeny. Aquaculture 197: 3-24.

30. Shin HS, Yoo JH, Min TS, Lee KY, Choi CY. 2010. The effects of quercetin on physiological characteristics and oxidative stress resistance in olive flounder, Paralichthys olivaceus. Asian-Australasian J Anim Sci 23: 588-597.

31. Slater CH, Schreck CB, Amend DF. 1995. GnRHa injection accelerates final maturation and ovulation/spermiation of sockeye salmon (Oncorhynchus nerka) in both fresh and salt water. Aquaculture 130: 279-285.

32. Zohar Y, Mylonas CC. 2001. Endocrine manipulations of spawning in cultured fish: from hormones to genes. Aquaculture 197: 99-136. 